\title{
CRITICAL POINTS ON CLOSED ELLIPTIC AFFINE SUBSPACES ${ }^{1,2}$
}

\section{ROBERT DELVER}

ABSTRACT. The critical points of a function restricted to the solution set of a linear elliptic equation are characterized. An extension of the Lagrange multiplier method is given. Existence and the relation to elliptic eigenvalue problems are discussed.

1. Introduction. In this paper, a multiple-integral variational problem with linear elliptic side condition is treated.

In $\$ 2$ Banach's closed range theorem is used to derive the equation of the critical points of a function defined on a Banach space $X$, restricted to the pre-image of an element in the range of a closed linear operator with closed range. This leads to an extension of the Lagrange multiplier method.

Let $J: H^{l}(\Omega) \rightarrow R$ be the multiple-integral function, and $L: H^{2 m}(\Omega) \rightarrow$ $H^{0}(\Omega)$ the elliptic operator. In $\$ 3$, the closed range property of the closure of $L$ in $H^{l}(\Omega)$ is proved, and after that the theory of $\$ 2$ is applied. In $\$ 4$, the resulting critical point equation is analyzed and in $\$ 5$ the existence of critical points is discussed.

In this note, earlier results of the writer [3], [4] are strengthened while the proofs are much simpler. Also the relation to work of other writers has become clear. Besides the relation to $L$. A. Ljusternik's Banach space version of the Lagrange multiplier method there is one to the elliptic eigenvalue problems studied by F. E. Browder [1]. The critical point equation in the present note is of the form

$$
J^{\prime}(u)=\lambda \circ \bar{L},
$$

where $J^{\prime}(u) \in \mathfrak{L}(\mathscr{D}(\bar{L}), R), \lambda \in \mathfrak{L}\left(H^{0}(\Omega), R\right), \bar{L}$ is the closure of $L, \mathscr{D}(\bar{L})$ is

Received by the editors June 11, 1974.

AMS (MOS) subject classifications (1970). Primary 35J35, 47A05, 58E15; Secondary 49A20, 49B25.

Key words and phrases. Variational problems, critical points, Lagrange multipliers, variational adjoint, elliptic boundary control problems, elliptic operatoreigenvalue problems.

1 The preparation of this paper was partially supported by the National Research Council under NRC grant A8731.

2 Most of the work was done while the writer held a visiting position at the Rijksuniversiteit te Groningen, The Netherlands. 
the domain of $\bar{L}$. This equation can be regarded as an elliptic operator eigenvalue problem. An explicit existence result for an eigenfunction-eigenvalue pair is given in the application in $\$ 5$.

The problem studied in this paper arises naturally in the optimal control at the boundary of systems governed by elliptic partial differential equations.

2. Abstract theory. Let $X$ and $Y$ be Banach spaces, $T: \mathcal{D}(T) \subset X \rightarrow Y$ a closed linear operator such that $\mathscr{D}(T)$ is dense in $X$, and the image $\mathscr{R}(T)$ is closed in $Y$. For a given $y \in R(T), T^{-1} y=\{x \in X: T x=y\}$. Let $f$ be a given differentiable function from $\mathscr{D}(f) \subset X$ into $R$ such that $\mathscr{D}(f)$ is open in $X$ and $T^{-1} y \subset \mathscr{D}(f)$. $\mathscr{L}(X, Y)$ denotes the space of continuous linear functions on $X$ into $Y$. For $g \in X^{\prime}=\mathfrak{L}(X, R)$, a $\in X$, let $\langle a, g\rangle_{X}$ denote $g(a)$.

Theorem 2.1. The function $f$ restricted to $T^{-1} y$ has a critical point at $x \in T^{-1} y$ iff there exists a $\lambda \in Y^{\prime}$ such that

$$
\left\langle h, f^{\prime}(x)\right\rangle_{X}=\langle T h, \lambda\rangle_{Y}, \forall h \in \mathscr{D}(T) .
$$

Proof. As $\mathscr{D}(T)$ is dense in $X$ the adjoint $T^{*}: \mathscr{D}\left(T^{*}\right) \subset Y^{\prime} \rightarrow X^{\prime}$ is well defined. As $T$ is a closed operator with closed range, $\mathcal{R}\left(T^{*}\right)=\mathcal{K}(T)^{\perp}$, by the closed range theorem (see e.g. [12]), where $\mathcal{K}(T)=\{x \in X: T x=0\}$.

$x$ is a critical point of $f$ restricted to $T^{-1} y \Leftrightarrow\left\langle h, f^{\prime}(x)\right\rangle=0, \forall b \in$ $\mathcal{K}(T) \Leftrightarrow \exists \lambda \in Y^{\prime}$ such that $f^{\prime}(x)=T^{*} \lambda \Leftrightarrow\langle T h, \lambda\rangle=\left\langle h, f^{\prime}(x)\right\rangle, \forall b \in \mathcal{D}(T)$.

If $\lambda \in Y^{\prime}$ is such that $\lambda \circ T$ is continuous on $\mathcal{D}(T)$ then let $\lambda \approx T$ denote its unique continuous linear extension over $X$ restricted to $\mathscr{D}(f)$.

Corollary. $x$ is a critical point of $f$ restricted to $T^{-1} y$ iff there exists a $\lambda \in Y^{\prime}$ such that $\lambda \circ T$ is continuous on $\mathscr{D}(T)$ and $x$ is a critical point of $f-\lambda \approx T$.

Proof. Let $x$ be a critical point of $f$ restricted to $T^{-1} y$; then by Theorem 2.1, there exists a $\lambda \in Y^{\prime}$ such that $\lambda \circ T$ is continuous and $\left(f^{\prime}(x)-\right.$

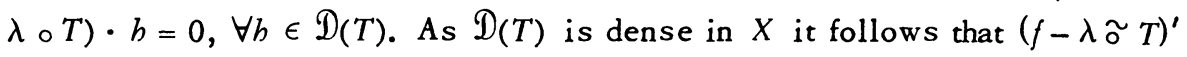
vanishes at $x$. The converse is trivial.

The Corollary shows the precise relation between the theorem and the Banach space formulation of the method of Lagrange multipliers, see e.g. [5]. The theorem reduces to this method if $T$ is continuous. If $T$ is not continuous the side condition $T x=y$ is not differentiable in which case the Lagrange multiplier rule, as it was known, cannot be used.

3. Elliptic variational problems. In this section the abstract theory will be used to characterize the critical points of a function defined in a neigh- 
bourhood of a closed elliptic affine subspace restricted to that set. First these affine subspaces will be defined. For simplicity it will be assumed that the data are smooth.

$\Omega$ will always denote an open bounded domain in $R^{n}$ with smooth boundary $\partial \Omega$. A uniformly strongly elliptic differential polynomial $A$ of order $2 m$ (see e.g. [6]) is given by

$$
(A u)(x)=\sum_{|\alpha| \leq 2 m} a_{\alpha}(x) D^{\alpha} u(x), \quad x \in \Omega,
$$

for all $u \in C^{2 m}(\Omega)$. $a_{\alpha} \in C^{\infty}(\bar{\Omega}), 0 \leq|\alpha| \leq 2 m$. For nonnegative integers $j$ let $H^{j}(\Omega)$ denote the Sobolev space $H_{2}^{j}(\Omega)$ or $W_{2}^{j}(\Omega)$. For $0 \leq k \leq 2 m$ let $L_{k}$ denote the linear operator from $H^{k}(\Omega)$ into $H^{0}(\Omega)$ with domain $\mathscr{D}\left(L_{k}\right)=$ $H^{2 m}(\Omega)$ given by

$$
\left(L_{k} u\right)(x)=(A u)(x), \quad x \in \Omega, \quad u \in H^{2 m}(\Omega),
$$

where the derivatives must be taken in $H^{2 m}$-sense.

Each of the operators $L_{k}$ is closable. For $k=0$, this is shown for example in [12]. For $1 \leq k \leq 2 m$ the proof is similar. Let $\bar{L}_{k}$ denote the closure of $L_{k}$.

Definition. A closed elliptic affine subspace is a set $U_{l, f}$ given by

$$
U_{l, f}=\left\{u \in D\left(\bar{L}_{l}\right): \bar{L}_{l} u=f\right\} \text {, }
$$

where $l \in\{0, \ldots, 2 m\}$ and $f \in R\left(\bar{L}_{l}\right)$ are given. $H^{l}(\Omega)$.

As $\bar{L}_{l}$ is a closed linear operator, $U_{l, f}$ is a closed affine subspace of

Lemma 3.1. $R\left(\bar{L}_{k}\right)$ is closed, $0 \leq k \leq 2 m$, and $\Re_{\left(L_{2 m}\right)=} R\left(\bar{L}_{k}\right), 0 \leq$ $k \leq 2 m$.

Proof. The closing operation of $L_{k}$ is defined by: $\bar{L}_{k} u=v \Leftrightarrow \exists\left\{u_{n}\right\} \subset$ $H^{2 m}$ such that $s-H^{k}-\lim _{n \rightarrow \infty} u_{n}=u$ and $s-H^{0}-\lim _{n \rightarrow \infty} A u_{n}=v$. Hence,

$$
\Re\left(L_{2 m}\right)=R\left(\bar{L}_{2 m}\right) \subset \cdots \subset \mathfrak{R}\left(\bar{L}_{0}\right) \subset \overline{R\left(L_{2 m}\right)} \text {, }
$$

so that it suffices to show that $R\left(L_{2 m}\right)$ is closed in $H^{0}(\Omega)$.

It is well known that there exists a finite dimensional subspace $P$ of $H^{0}(\Omega)$ such that $A u=f, u \in H^{2 m}(\Omega) \cap H_{0}^{m}(\Omega)$ is solvable iff $f \in P^{\perp}$ (see e.g. [6, part I, Theorem 14.6, Theorem 17.2]). Put $\left.Q=R_{\left(L_{2 m}\right.}\right) \cap P$ then $R\left(L_{2 m}\right)=P^{\perp}+Q$. As $P^{\perp}$ and $Q$ are closed orthogonal sets $\Re_{\left(L_{2 m}\right)}$ is closed. 
Let a function $J: H^{l} \rightarrow R$ be given by

$$
J(u)=\int_{\Omega} F\left(x, u(x), D u(x), \ldots, D^{l} u(x)\right) d x .
$$

Here $F=F(x, \xi): \Omega \times R^{N} \rightarrow R$ is measurable in $x$ and differentiable with respect to the other variables. $D^{k} u$ denotes all derivatives of $u$ of order $k$. Assume that $J: H^{l} \rightarrow R$ is differentiable in an open neighbourhood of $U_{l, f}$. The differentiability can be assured by imposing growth conditions on $F$ as was done for example in F. E. Browder's paper [2]. In this case

$$
J^{\prime}(u) \cdot w=\sum_{|\alpha| \leq l}\left(F_{\alpha} u, D^{\alpha} w\right),
$$

where $($,$) denotes the H^{0}(\Omega)$ scalar product and

$$
F_{\alpha} u=\partial^{|a|} F\left(x, u, \ldots, D^{l} u\right) / \partial\left(D^{a} u\right)
$$

As each of the operators $\bar{L}_{l}$ satisfies the conditions imposed on the operator $T$ of $\$ 2$, the abstract theory can be applied. Theorem 2.1 takes the form:

Theorem 3.1. $u \in U_{l, f}$ is a critical point of $J$ restricted to $U_{l, f}$ iff there exists a $\lambda \in\left(H^{0}(\Omega), R\right)$ such that

$$
J^{\prime}(u) \cdot w=\lambda \circ \bar{L}_{l} \cdot w, \quad \forall w \in \mathfrak{M}\left(\bar{L}_{l}\right) .
$$

If $l=2 m$ this result could be obtained from the Lagrange multiplier method.

Let $n$ denote the natural isometry: $\mathcal{L}\left(H^{0}(\Omega), R\right) \rightarrow H^{0}(\Omega)$ and put $v=$ $n(\lambda)$. As $C^{\infty}(\bar{\Omega})$ is dense in $\mathscr{D}\left(\bar{L}_{l}\right),(3.8)$ is equivalent to

$$
\int_{\Omega} \sum_{|\alpha| \leq l} F_{\alpha} u D^{\alpha} \psi d x=\int_{\Omega} v A \psi d x, \quad \forall \psi \in C^{\infty}(\bar{\Omega}) .
$$

In [3], [4], a function $v \in C^{2 m}(\bar{\Omega})$ satisfying (3.9) was called a variational adjoint. In this case (3.9) was shown to be equivalent to a boundary value problem called the variational boundary value problem. The relation (3.9) or (3.8) is a generalized version thereof. The function $v$ is still called a variational adjoint.

Application. Let $A=\Delta=$ the Laplacian in $R^{n}$ and let $J: H^{1}(\Omega) \rightarrow R$ be given by $J(u)=1 / 2(\nabla u, \nabla u)$ where $\nabla=\left(\partial / \partial x_{1}, \ldots, \partial / \partial x_{n}\right)$ (Dirichlet integral). $f$ is a given element of $H^{0}(\Omega)$. (3.9) takes the form

$$
(\nabla u, \nabla \psi)=(\nu, \Delta \psi), \quad \forall \psi \in C^{\infty}(\Omega) \text {, some } v \in H^{0}(\Omega) \text {. }
$$


Restricting $\psi$ to the harmonic functions and integrating the left-hand side by parts one obtains

$$
\int_{\partial \boldsymbol{\Omega}} \tilde{u} \frac{\partial \psi}{\partial \mathrm{n}} d x=0
$$

here $\tilde{u} \in H^{0}(\partial \Omega)$ is the trace of $u$ on $\partial \Omega$. The function $\partial \psi / \partial \mathrm{n}$ can be chosen freely as long as $\int \partial \psi / \partial \mathbf{n} d x=0$ so that $\tilde{u}$ is constant a.e., by the lemma of Dubois-Raymond.

Conversely, if $u \in H^{1}(\Omega)$ has constant trace on $\partial \Omega$ one observes that

$$
(\nabla u, \nabla \psi)_{\mathbf{\Omega}}=-(u, \Delta \psi)_{\mathbf{\Omega}}+(c, \partial \psi / \partial \mathbf{n})_{\partial \boldsymbol{\Omega}}=(c-u, \Delta \psi)_{\mathbf{\Omega}},
$$

so that (3.10) is satisfied with $v=c-u$.

In consequence, the critical set of $J$ restricted to $U_{1, f}$ consists precisely of those elements with constant trace on $\partial \Omega$.

One easily proves that $J$ assumes its infimum over $U_{1, f}$ in each of the critical points.

An analogous physical problem occurs if one tries to fix a loaded membrane in a vertical cylinder $\Omega \times R$ along the lateral boundary $\partial \Omega \times R$ in such a way that the membrane takes on the flattest possible shape (given load and cylinder), where flatness is measured by the Dirichlet integral. It happens that one should fix the membrane along a horizontal curve.

A somewhat weaker form was given in [3].

4. Analysis of the critical point equation. In this section some properties of pairs $u, v$ satisfying (3.9) will be derived.

Lemma 4.1. Let $A$ and $J$ be as in $\S 3$, let $f \in R\left(\bar{L}_{l}\right) \cap H^{k}(\Omega)$ be given and let $u \in U_{l, f}, v \in H^{0}(\Omega)$ be such that (3.9) is satisfied, then $u \epsilon$ $H_{10 \mathrm{c}}^{2 m+k}(\Omega), v \in H_{10 \mathrm{c}}^{4 m-2 l+k}(\Omega)$ and $v$ is a weak solution of

$$
{ }^{t} A v=[F], u, \quad x \in \Omega^{\prime} \subset \Omega, \quad \bar{\Omega}^{\prime} \subset \Omega,
$$

where ${ }^{t} A$ is the formal adjoint of $A$ and $[F], u \in H_{10 c}^{2 m-2 l+k}(\Omega)$ is given by

$$
[F]_{, u}=\sum_{|a|<l}(-1)^{|a|} D^{a} F_{a} u .
$$

Proof. For any $u \in U_{l, f}$ there exists a sequence $\left\{u_{n}\right\} \subset H^{2 m}(\Omega)$ such that $s-H^{l}-\lim _{n \rightarrow \infty}, u_{n}=u$ and $s-H^{0}-\lim _{n \rightarrow \infty} A u_{n}=f$. For each $u_{n}$ :

$$
\left(A u_{n}, \phi\right)=\left(u_{n},{ }^{t} A \phi\right), \quad \forall \phi \in C_{0}^{\infty} \text {. }
$$

Letting $n \rightarrow \infty$ it follows that $u$ is a weak solution of $A u=f$, so that 
$u \in H_{10 \mathrm{co}}^{2 m+k}(\Omega)$ by Friedrich's theorem (see for example [12]).

From (3.9), restricting $\psi$ to $C_{0}^{\infty}(\Omega)$ and integrating by parts, it follows that $v$ is a weak solution of (4.1), so that again by Friedrich's theorem $v \epsilon$

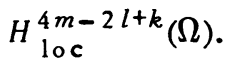

The explicit variational adjoint boundary conditions given in [3], [4], can only be justified if $u \in H^{2 l}(\Omega)$, while no reason for this seems to exist except of course if $l=0$.

Lemma $4.2(F=F(x, u))$. Let $A, J, f, u, v$, be as in Lemma 4.1, with $l=0$. Then (3.9) is equivalent with

$$
\begin{gathered}
u \in H_{\mathrm{loc}}^{2 m}(\Omega), \quad v \in H_{0}^{2 m}(\Omega), \\
{ }^{t} A v=\partial F / \partial u, \quad x \in \Omega .
\end{gathered}
$$

Proof. As $A$ is uniformly strongly elliptic on $\Omega$ there exists an open bounded domain $\Omega^{+}, \bar{\Omega} \subset \Omega^{+}$and an extension $\tilde{A}$ of $A$ in $\Omega^{+}$such that $\tilde{A}$ is uniformly strongly elliptic on $\Omega^{+}$. Let $\partial F / \partial u$ and $\widetilde{v}$ denote the extension by zero into $\Omega^{+}$of $\partial F / \partial u$ and $v$ respectively, then by (3.9):

$$
(\partial F / \partial u, \phi)_{\mathbf{Q}^{+}}=(\tilde{v}, \tilde{A} \phi)_{\mathbf{Q}^{+}}, \quad \forall \phi \in C_{0}^{\infty}\left(\Omega^{+}\right)
$$

so that $\tilde{v}$ is a weak solution of ${ }^{t} A v=\partial F \tilde{/} \partial u$, and as $\partial F / \partial u \in H^{0}\left(\Omega^{+}\right)$it follows that $\widetilde{v} \in H_{\text {loc }}^{2 m}\left(\Omega^{+}\right)$. Hence $v \in H^{2 m}(\Omega)$ and as the traces of $v$ on $\partial \Omega$ all vanish $v \in H_{0}^{2 m}(\Omega)$ (see e.g. [8]).

5. Remarks on existence. Since $U_{l, f}$ is a closed affine subspace of $H^{l}(\Omega)$ several known methods can be used to obtain existence results. The essential reason for working with $\bar{L}_{l}$ instead of $L_{2 m}$, in which case the Lagrange multiplier method could be used directly, is that results like $|J(u)|$ $\rightarrow \infty$ as $\|u\| \rightarrow \infty$ or coercivity of $J$ over Sobolev spaces of higher order than the order of differentiation in the integrand of $J$ cannot be obtained.

(a) Lower semicontinuity. As $U_{l, f}$ is strongly closed and convex it is weakly closed. Thus if: (1) $J$ is bounded below on $U$; (2) $|J(u)| \rightarrow \infty$ as $\|u\|_{H} l \rightarrow \infty, u \in U_{l, f} ;$ (3) $J$ is weakly sequentially lower semicontinuous on $U_{l, f}$; then $J$ restricted to $U_{l, f}$ assumes its infimum. If in addition $J$ is differentiable then there exists at least one pair $u \in U_{l, f}, v \in H^{0}(\Omega)$, such that (3.9) is satisfied.

Conditions on the integrand of $J$ ensuring the above properties can be found for example in F. E. Browder [1], [2] and in C. B. Morrey [9]. 
(b) The Palais-Smale condition. As $U_{l, f}$ is a complete Riemannian manifold in $H^{l}(\Omega)$, Ljusternik-Schnirelman and Morse theory can be applied if $J$ satisfies the P.S.-condition; see [10] and [11]. In particular if $J$ restricted to $U_{l, f}$ is bounded below and satisfies the P.S.-condition then it assumes its infimum.

Example. Let $A$ be given by (3.1) with $m=1$, let $f \in R\left(\bar{L}_{1}\right)$. Let a bilinear form $B$ on $H^{1}(\Omega)$ be given by

$$
B(u, v)=\int_{\Omega}\left\{\sum_{|a|,|\beta|=1} b_{\alpha \beta} D^{a} u D^{\beta} v+b u v\right\} d x
$$

with $b_{a \beta} \in C(\bar{\Omega}), b \in C(\bar{\Omega})$. Further, let there exist a positive constant $\beta$ such that

$$
\begin{gathered}
\sum_{|\alpha|,|\beta|=1} b_{\alpha \beta}(x) \xi_{\alpha} \xi_{\beta} \geq \beta|\xi|^{2}, \quad \forall x \in \Omega, \xi \in R^{n} \\
b(x) \geq \beta, \quad \forall x \in \Omega,
\end{gathered}
$$

then $B(u, u) \geq \beta\left(\|u\|_{H^{1}}\right)^{2}$. Put $J(u)=B(u, u)$.

It is easy to show (see for example J. L. Lions [7, Chapter 1]), that J is differentiable in a neighbourhood of $U_{1, f}$ and that (1), (2), (3) of (a) are satisfied. As $J$ is strictly convex there is only one critical point $u$. From Theorem 3.1 it then follows that there is precisely one $u \in U_{1, f}$ such that

$$
2 B(u, \psi)=(v, A \psi), \quad \forall \psi \in C^{\infty}(\bar{\Omega}),
$$

is solvable for some $v \in H^{0}(\Omega)$.

\section{BIBLIOGRAPHY}

1. F. E. Browder, Variational methods for nonlinear elliptic eigenvalue problems, Bull. Amer. Math. Soc. 71 (1965), 176-183. MR 31 \#3707.

2. - Remarks on the direct method of the calculus of variations, Arch. Rational Mech. Anal. 20 (1965), 251-258. MR 32 \#4576.

3. R. Delver, Variational problems within the class of solutions of a partial differential equation, Trans. Amer. Math. Soc. 180 (1973), 265-289.

4. - Boundary and interior control for partial differential equations, Canad. J. Math. 27 (1975), 200-217.

5. —_, Elliptic variational problems. II, Lecture Notes, Rijksuniversiteit Groningen, 1974. (Available on request.)

6. A. Friedman, Partial differential equations, Holt, Rinehart and Winston, New York, 1969.

7. J.-L. Lions, Controle optimal de systèmes gouvermés par des équations aux dérivées partielles, Dunod; Gauthier-Villars, Paris, 1968; English transl., Die Grundlehren der math. Wissenschaften, Band 170, Springer-Verlag, Berlin and New York, 1971. MR 39 \#5930; MR 42 \#6395.

8. J.-L. Lions and E. Magenes, Problèmes aux limites non homogènes et ap- 
plications. Vol. I, Travaux et Recherches Mathématiques, no. 17, Dunod, Paris, 1968. MR $40 \# 512$.

9. C. B. Morrey, Jr., Multiple integrals in the calculus of variations, Die Grundlehren der math. Wissenschaften, Band 130, Springer-Verlag, New York, 1966. MR 34 $\# 2380$.

10. R. S. Palais and S. Smale, A generalized Morse theory, Bull. Amer. Math. Soc. 70 (1964), 165-172. MR 28 \#1634.

11. J. T. Schwartz, Generalizing the Lusternik-Schnirelman theory of critical points, Comm. Pure Appl. Math. 17 (1964), 307-315. MR 29 \#4069.

12. K. Yosida, Functional analysis, 3rd ed., Springer-Verlag, New York, 1971.

DEPARTMENT OF MATHEMATICS, UNIVERSITY OF TORONTO, TORONTO, ONTARIO, CANADA 